\title{
Dysplasie ectodermique : proposition de prise en charge prothétique
}

\section{RÉSUMÉ}

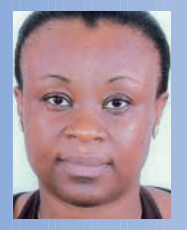

Yomin Cécile ALLOH AMICHIA Docteur en chirurgie dentaire, Maître-assistant

Faculté de chirurgie dentaire d'Abidjan, Assistante associée des Universités Faculté de Chirurgie Dentaire de Nantes, Département de Prothèses, 1, place Alexis Ricordeau, BP 84215,

44042 Nantes Cedex 01.

Aboudramane BAMBA

Docteur en chirurgie dentaire,

Maître de conférence agrégé

Faculté de chirurgie dentaire d'Abidjan.

Département de Prothèses,

22 BP 612 ,

Abidjan 22. Côte d'Ivoire.

\section{Laurent LE GUÉHENNEC}

Docteur en chirurgie dentaire, MCU-PH

Faculté de chirurgie dentaire de Nantes.

Département de Prothèses,

1, place Alexis Ricordeau,

BP 84215 ,

44042 Nantes Cedex 01.

\section{Pascal BOURDEAUT}

Docteur en chirurgie dentaire, Spécialiste qualifié

en orthopédie dento-faciale,

10 place François Blancho,

44600 Saint-Nazaire.

\section{Bernard GIUMELLI}

Docteur en chirurgie dentaire,

PU-PH,

Faculté de chirurgie dentaire de Nantes,

Département de Prothèses,

1, place Alexis Ricordeau,

BP 84215,

44042 Nantes Cedex 01.
La dysplasie ectodermique anhidrotique, liée au chromosome $X$, est la forme la plus fréquente des dysplasies ectodermiques. Elle se manifeste par une triade de signes : une hypohidrose ou anhidrose (atteinte des glandes sudoripares et sébacées), une hypotrichose (atteinte des cheveux, des poils), une hypodontie ou anodontie (diminution partielle ou totale du nombre de dents).

L'absence des dents constitue un handicap fonctionnel et esthétique, relevant d'une thérapeutique prothétique. Pour des raisons psychosociales, cette réhabilitation est nécessaire dès le jeune âge.

Cet article décrit les différentes étapes prothétiques d'une réhabilitation orale, chez un jeune homme âgé de 25 ans, atteint de dysplasie ectodermique de type Christ-Siemens-Touraine. hypohidrose ou anhidrose

- hypotrichose

réhabilitation prothétique

\section{(C) AEOS / EDP Sciences}




\section{Introduction}

$>$ La dysplasie ectodermique (DE) anhidrotique ou syndrome de Christ-SiemensTouraine fait partie du groupe hétérogène des dysplasies ectodermiques qui touchent les organes d'origine ectodermique ainsi que leurs dérivés. Au cours des dix dernières années, plus de 170 entités pathologiques ont été décrites comme $\mathrm{DE}$, toutes partageant une anomalie primaire du poil, de la dent, de l'ongle et de la glande sudoripare eccrine $[1,2,22,23,28]$. Beaucoup sont associées à des anomalies d'autres organes ou systèmes et s'accompagnent parfois d'un retard mental. Les anomalies concernant l'épiderme et ses annexes sont très variables et une superposition clinique existe parmi la plupart des DE [17].

La dysplasie ectodermique anhidrotique est liée au chromosome $X$. Elle représente la forme la plus connue et la plus fréquente des DE (80\% des DE selon I'association française des dysplasies ectodermiques). Sa prévalence est de 1/100 000 naissances $[9,16,20,2226]$. Les deux sexes peuvent être atteints avec une prédominance masculine en rapport avec le mode de transmission le plus souvent récessif $[2,11,26]$. Cette dysplasie se retrouve dans toutes les races et sur tous les continents. Ce syndrome est caractérisé par une triade de signes $[4,5,9$, $10,12,16,18,22,26]$ : hypohidrose ou anhidrose, hypotrichose, hypodontie ou anodontie. L'hypohidrose ou anhidrose est caractérisée par l'absence de glandes sudoripares et sébacées, ce qui entraîne une absence de sudation, ou tout au moins sa diminution importante avec un risque d'hyperthermie maligne. L'hypotrichose se manifeste par des cheveux secs très clairsemés et une raréfaction dans les poils. L'hypodontie ou anodontie se caractérise par l'absence de toutes ou presque toutes les dents. Les dents présentes ont le plus souvent une morphologie anormale (dents coniques, conoïdes, riziformes au niveau incisif mais aussi au niveau canin et prémolaire). Le plus souvent, seules les premières molaires définitives supérieures et inférieures ainsi que les incisives centrales supérieures sont présentes alors que les prémolaires supérieures et les incisives inférieures sont le plus souvent absentes. Ces dysmorphoses touchent aussi bien la première et la seconde dentition. Les parties anodontiques des maxillaires ne forment pas de procès alvéolaires, raison pour laquelle la croissance verticale des maxillaires est entravée [15]. Des aplasies des crêtes alvéolaires sont aussi fréquemment rencontrées. L'hypodontie conduit à des troubles de l'articulé avec modifications des rapports osseux.

Des complications générales peuvent être liées à ce syndrome, telles que des atteintes oculaires et respiratoires et un retard intellectuel et staturo-pondéral. Les signes cliniques caractéristiques sont une peau sèche, une rhinite atrophique avec un nez en selle, la proéminence des arcades sourcilières et du menton, des troubles des ongles, des lèvres épaisses et éversées, de larges oreilles.

Le diagnostic prénatal porte sur la révélation de l'absence de glandes sudorales sur les biopsies cutanées fœtales obtenues sous fœtoscopie à la $20^{\mathrm{e}}$ semaine. Plus récemment, l'identification des mutations responsables par I'utilisation de marqueurs génétiques permet de réaliser un diagnostic prénatal à partir de trophoblaste à 9 semaines de grossesse.

Il semble n'exister à l'heure actuelle aucun traitement curatif ou causal. La prise en charge n'est que symptomatique [3]. Elle vise à éviter I'hyperthermie chez le nourrisson et l'enfant. Le traitement est ensuite multidisciplinaire afin de pallier les différents troubles observés. Au niveau dentaire, ce traitement doit être précoce et débute généralement vers 3 ans [11, 22]. 
La réhabilitation dentaire par prothèse adjointe ou conjointe rétablit la fonction et l'esthétique et contribue ainsi au développement staturo-pondéral et psychoaffectif de l'enfant. Tout type de prothèse (fixée, amovible ou implanto-portée) est envisageable pour la réhabilitation dentaire, en fonction de l'âge et du développement du patient. Toutefois des problèmes relatifs au nombre de piliers insuffisants présentant en plus des pulpes volumineuses et des couronnes cliniques courtes avec peu de rétention rendent les réhabilitations par prothèse fixée difficiles. Pour ces raisons, la majorité des jeunes patients sont réhabilités au plan dentaire par des prothèses amovibles.

La réhabilitation par prothèse implanto-portée ne concerne le plus souvent que les patients ayant terminé leur croissance (soit 15-16 ans chez les filles et 17-18 ans chez les garçons). La mise en place d'implants repose sur l'analyse de la maturité dentaire et squelettique plus que sur l'âge chronologique. En effet, les implants placés au maxillaire avant la fin de la croissance ont un pronostic réservé. Ils se comportent comme des dents ankylosées et ne peuvent bouger ou s'adapter aux modifications osseuses avec un risque d'infraclusion. De même, la solidarisation de plusieurs implants est aussi susceptible d'entraver la croissance ultérieure. La pose des implants chez l'enfant reste donc très controversée du fait de la croissance, à l'exception des implants antérieurs mandibulaires chez les enfants atteints d'anodontie (vers l'âge de 56 ans) ou des cas sévères d'hypodontie. Pour ces patients, les implants semblent présenter un vrai challenge malgré un taux d'échec élevé [6]. Des implants ont même été posés avec succès chez un enfant de 30 mois dans la région antérieure mandibulaire [7]. Néanmoins, ces jeunes patients doivent bénéficier d'un suivi régulier incluant des analyses céphalométriques. L'utilisation des implants apporte de réelles satisfactions sur le plan fonctionnel, psychosocial $[16,22,23,25]$. La décision d'implanter doit être pluridisciplinaire. Le degré d'hypodontie, les troubles psychosociaux relatifs, le nombre et le site des dents absentes doivent aussi être pris en compte [16, 23, 24].

Cet article illustre la planification et la réalisation d'une nouvelle réhabilitation prothétique complexe chez un patient actuellement âgé de 25 ans atteint de dysplasie ectodermique avec hypodontie.

\section{Historique de la prise en charge pluridisciplinaire de la naissance à la première prothèse} à 16 ans

La prise en charge de ce syndrome de ChristSiemens-Touraine a été pluridisciplinaire. Elle a débuté vers l'âge de 1 an avec des consultations pédiatriques répétées devant l'inquiétude grandissante de la mère face aux retards d'éruption des dents temporaires et plus tard à la forme atypique des canines. Le diagnostic précoce de $\mathrm{DE}$, établi à 2 ans et demi par un orthodontiste, a permis aux parents de mettre un nom sur la maladie de leur fils, une orientation thérapeutique et une prise en charge de I'enfant. 
Au niveau dentaire, différents auteurs $[4,5,8$ $10,12,13,15,17,18,20-22,26]$ s'accordent à dire que le traitement prothétique doit être le plus précoce possible, avant la scolarisation de I'enfant. En effet, l'atteinte dentaire perturbe gravement le développement psychique de l'enfant, peut freiner son développement scolaire et affecter de façon pathologique sa vie relationnelle. La pose de prothèse permet à l'enfant de se sentir "comme les autres», de manger normalement, de dormir mieux, de pouvoir présenter son visage sans provoquer de railleries. Les différents objectifs de cette réhabilitation dentaire sont multiples, à savoir restaurer les fonctions de mastication et de phonation, améliorer la déglutition, rétablir une dimension verticale normale et favoriser une croissance faciale normale. Tout ceci aboutit à une intégration sociale et notamment scolaire de l'enfant.

Chez ce patient, la prise en charge dentaire s'est faite vers l'âge de 10-11 ans. À cet âge, il présente une bonne hygiène buccale avec l'absence de caries, avec 8 dents maxillaires: 11, $21,13,23,55,65,16,26$ et 6 dents mandibulaires: 36, 37, 46, 47, 85, 43 est en début d'éruption. La reconstruction coronaire par composite des dents dysmorphiques du groupe incisivo-canin $(13,23)$ a redonné un sourire au patient qui était la risée de ses camarades à l'école du fait de l'absence de certaines dents, et notamment de ses incisives inférieures. Concernant les cheveux, ceux-ci, très fins depuis son jeune âge, se sont mis à chuter au fur et à mesure de sa croissance, surtout vers l'âge de 13 ans. Ce phénomène est apparu plus perturbateur pour l'équilibre du patient que le problème dentaire (fig. $1 \mathrm{a}, \mathbf{1} \mathrm{b}$, 1 c, fig. 2 a, 2 b).

Trois psychologues ont également été consultés mais le patient leur a toujours opposé un mutisme total. Refusant de se considérer comme un sujet malade, il ne voyait pas la nécessité $d$ 'une telle prise en charge.

Du fait de l'absence d'un grand nombre de dents entraînant un hypo-développement alvéolaire, une disclusion postérieure est apparue. Un traitement orthodontique a été entrepris à l'âge de 10 ans. La mise en place d'un quad'hélix* pendant six mois a permis de réaliser une expansion maxillaire corrigeant une endomaxillie supérieure gauche et l'inversé d'articulé qui en résultait. La rééducation linguale par un écran lingual a permis la correction de la béance antérieure et un bumper** a contrecarré l'aspiration de la lèvre inférieure et rééduqué le buccinateur. Enfin, un arc transpalatin et la pose de bagues au maxillaire ont permis de placer les incisives centrales et canines maxillaires en normoclusion et de symétriser les canines mandibulaires. Grâce à ce traitement orthodontique, l'arcade dentaire est préparée pour la réhabilitation prothétique (fig. 3 a et $\mathbf{3}$ b).

La phase prothétique est la dernière étape du traitement. Chez l'enfant et l'adolescent, le traitement de choix reste la réhabilitation par prothèse amovible afin de s'adapter à la croissance des maxillaires et aux futures éruptions dentaires. Chez ce patient, la solution retenue consiste en des prothèses adjointes amovibles de recouvrement à châssis métallique. Ces prothèses sur overdentures utilisant la technique des dents massives présentent l'avantage d'être plus stables et rétentives, esthétiquement satisfaisantes tout en conservant les organes dentaires et la sensibilité proprioceptive. Elles nécessitent par contre une hygiène

\footnotetext{
*quad'helix : dispositif mécanique soudé sur des bagues postérieures à proximité du palais osseux destiné à vestibuler les secteurs lateraux de l'arcade supérieure.

**bumper : dispositif mécanique destiné à annuler les forces musculaires de la sphère orale durant les dysfonctions.
} 

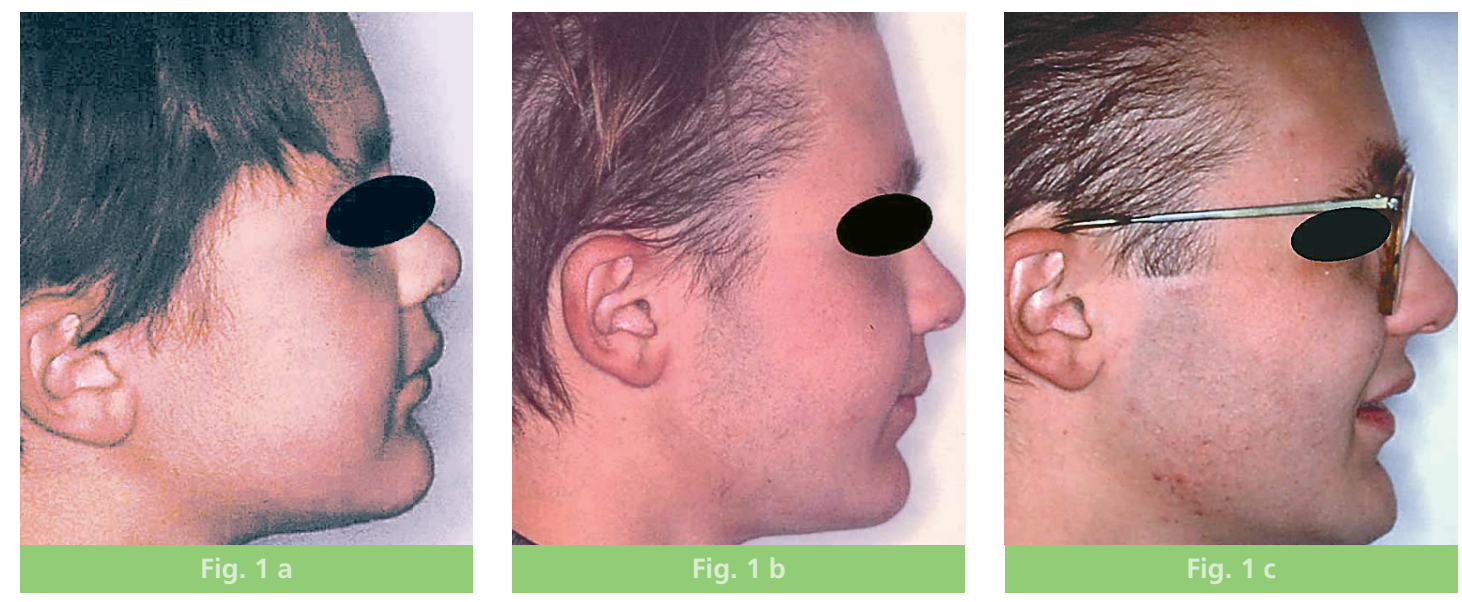

Fig. 1 a à $c$ Photos vues de profil. $a: 11$ ans ; $b: 13$ ans ; $: 16$ ans.
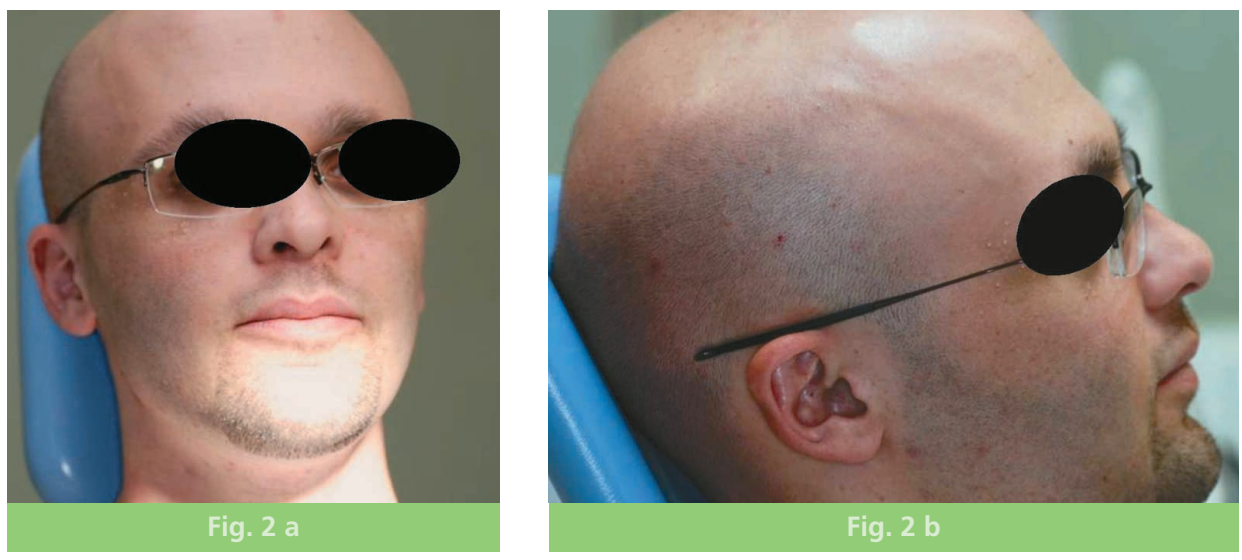

Fig. 2 a et $b$ Photos à 25 ans.

$a$ : vue de face ; $b$ : vue de profil.
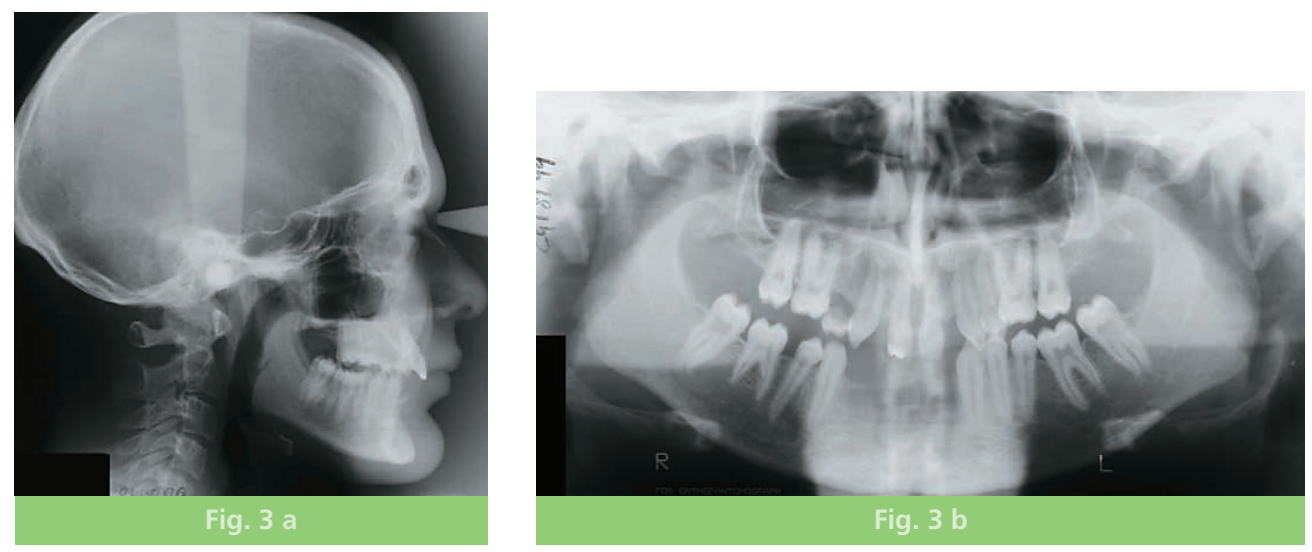

Fig. 3 a et b Situation initiale à 16 ans.

a : téléradiographie ; b : radiographie panoramique. 
rigoureuse $[3,9,13,19-21,27]$ et des contrôles très réguliers. Les prothèses ont été retouchées plusieurs fois lors des contrôles afin d'apporter les modifications indispensables en corrélation avec la croissance. Ces prothèses partielles amovibles à châssis coulé ont permis au patient de fonctionner sans problèmes majeurs et notamment de retrouver une alimentation normale. En effet, du fait de l'édentement partiel non compensé, le patient ne se nourrissait que d'aliments à la consistance molle.

\section{Deuxième prise en charge prothétique}

\section{à 25 ans}

\section{Anamnèse et examen clinique}

À l'âge de 25 ans, le patient nécessite une nouvelle réhabilitation prothétique du fait de I'usure de ses prothèses amovibles. Le patient est très réservé, n'aime pas trop parler de son affection et refuse de se considérer comme un malade. Toutefois, il s'est créé une relation de confiance avec son praticien qui favorise des rapports harmonieux. Très satisfait de ses prothèses en dépit de l'inadaptation due à leur ancienneté, il souffre surtout de son absence de cheveux avec un effet négatif sur ses relations affectives. De plus, de fréquentes douleurs sont dues à la présence d'aphtes.

L'examen met en évidence les signes cliniques de la dysplasie ectodermique : les cheveux sont clairsemés, les sourcils fins et la peau délicate et sèche du fait de la diminution du nombre des glandes sudoripares et sébacées. Le faciès est ovale, avec un profil légèrement concave. La DVO paraît adéquate. Le front est bombé (fig. $\mathbf{2}$ a et $\mathbf{2}$ b). L'examen endobuccal met en évidence I'hypoplasie des crêtes alvéolaires dans les régions édentées. Toutes les dents présentes se caractérisent par des morphologies atypiques, particulièrement les canines de formes conoïdes. Les couronnes des dents sont courtes, surtout au niveau des prémolaires et molaires. II existe une disclusion postérieure gauche (fig. 4 a à d). Les sillons des faces occlusales des molaires sont prononcés et nécessitent des obturations aux composites. L'hygiène bucco-dentaire est considérée comme satisfaisante. Les prothèses partielles amovibles à châssis coulé sont devenues inadaptées et ont été meulées à certains endroits.

Dix dents sont présentes au maxillaire $(11,12$, $13,16,17,21,23,25,26,27)$ et 9 à la mandibule $(33,34,35,36,37,43,44,46,47)$.

Le schéma dentaire se présente comme suit (fig. 3 b) :

X 1716 XX1312 11 $21 \times 23 \times 252627$ X X 3736353433 XX XX $4344 \times 4647$ X Les examens confirment les crêtes alvéolaires très étroites et de hauteur réduite. Même dans les régions dentées, le volume osseux est très réduit et les parois alvéolaires sont minces. En OIM, seuls 23 et 33 sont en contact et maintiennent la dimension verticale (fig. $\mathbf{4}$ a et $\mathbf{4}$ d). En conclusion, l'examen clinique met en évidence des morphologies dentaires présentant peu de rétention avec une inclinaison défavorable des dents antérieures maxillaires. Le volume osseux est insuffisant et s'oppose à la pose d'implants sans reconstruction préalable, notamment dans le secteur droit présentant un espace intermaxillaire trop important. 

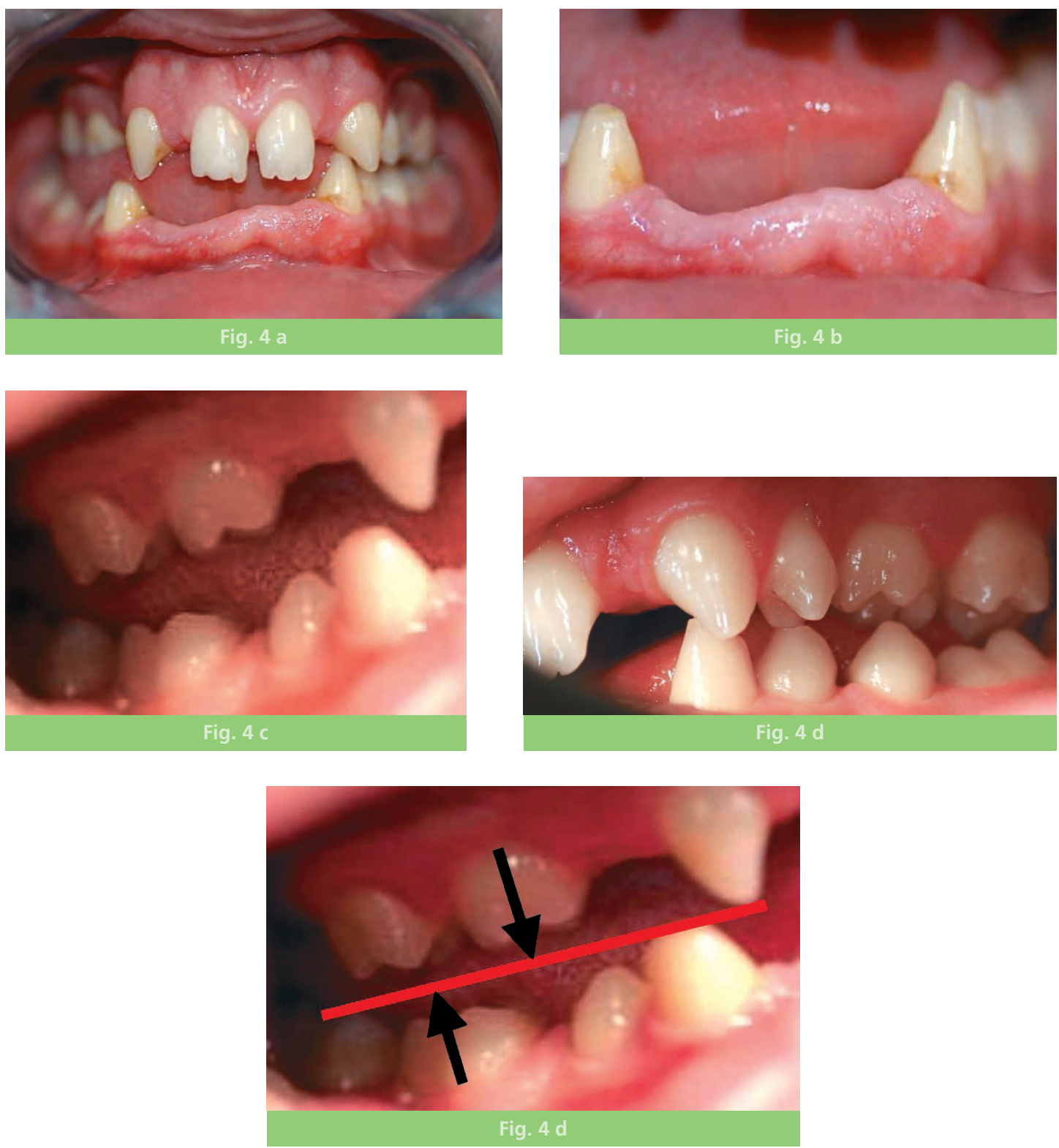

Fig. 4 a à e Situation initiale à 25 ans.

a : vue de l'occlusion de face; $b$ : arcade mandibulaire en vue de face ; $\mathbf{c}$ disclusion postérieure vue de profil droit ; $\mathbf{d}$ : disclusion postérieure vue de profil gauche et contact intercanin ; e : schématisation de la hauteur de couronne clinique très importante/ratio couronne-racine défavorable.

\section{Pronostic}

Du point de vue fonctionnel, il est préférable de réhabiliter le patient par des prothèses amovibles. En effet, les couronnes réalisées sur les dents postérieures mandibulaires afin de rétablir des contacts occlusaux présenteraient une hauteur de couronne clinique très importante avec un ratio couronne-racine défavorable (fig. 4 e). Au niveau des secteurs édentés, la réalisation de prothèses implanto-portées semble complexe du fait de 
la nécessité d'une reconstruction préalable des crêtes alvéolaires avant la mise en place d'implants. Le rapport hauteur de couronne clinique/longueur de l'implant serait là encore défavorable. En outre, au niveau antérieur mandibulaire, le soutien de la lèvre ne peut être obtenu sans fausse gencive. Seule une chirurgie des bases osseuses permettrait de rétablir une distance interarcade harmonieuse.

\section{Traitement}

Du fait de l'augmentation du risque carieux chez ce patient avec la prothèse amovible à recouvrement, la motivation à l'hygiène du patient est renforcée.

Une première série d'empreintes à l'alginate des deux arcades permet l'étude des modèles,
Aussi il est réalisé au maxillaire une prothèse adjointe à châssis métallique remplaçant 12,22 , une seule des deux prémolaires absentes, 24 ; l'espace existant étant trop réduit pour contenir plus d'une dent. À la mandibule, une prothèse amovible à recouvrement permet de remplacer les 31, 32, 33, 34 et la prémolaire 45 . Les dents postérieures $(44,46,47$ et $35,36,37)$ sont recouvertes afin de rétablir une dimension verticale satisfaisante.

notamment au paralléliseur, afin de déterminer l'axe d'insertion, le choix des crochets et le tracé des prothèses amovibles. Sur ces modèles, un porte-empreinte individuel (PEI) est réalisé. Après essayage, réglage et marginage des zones édentées, l'empreinte secon-
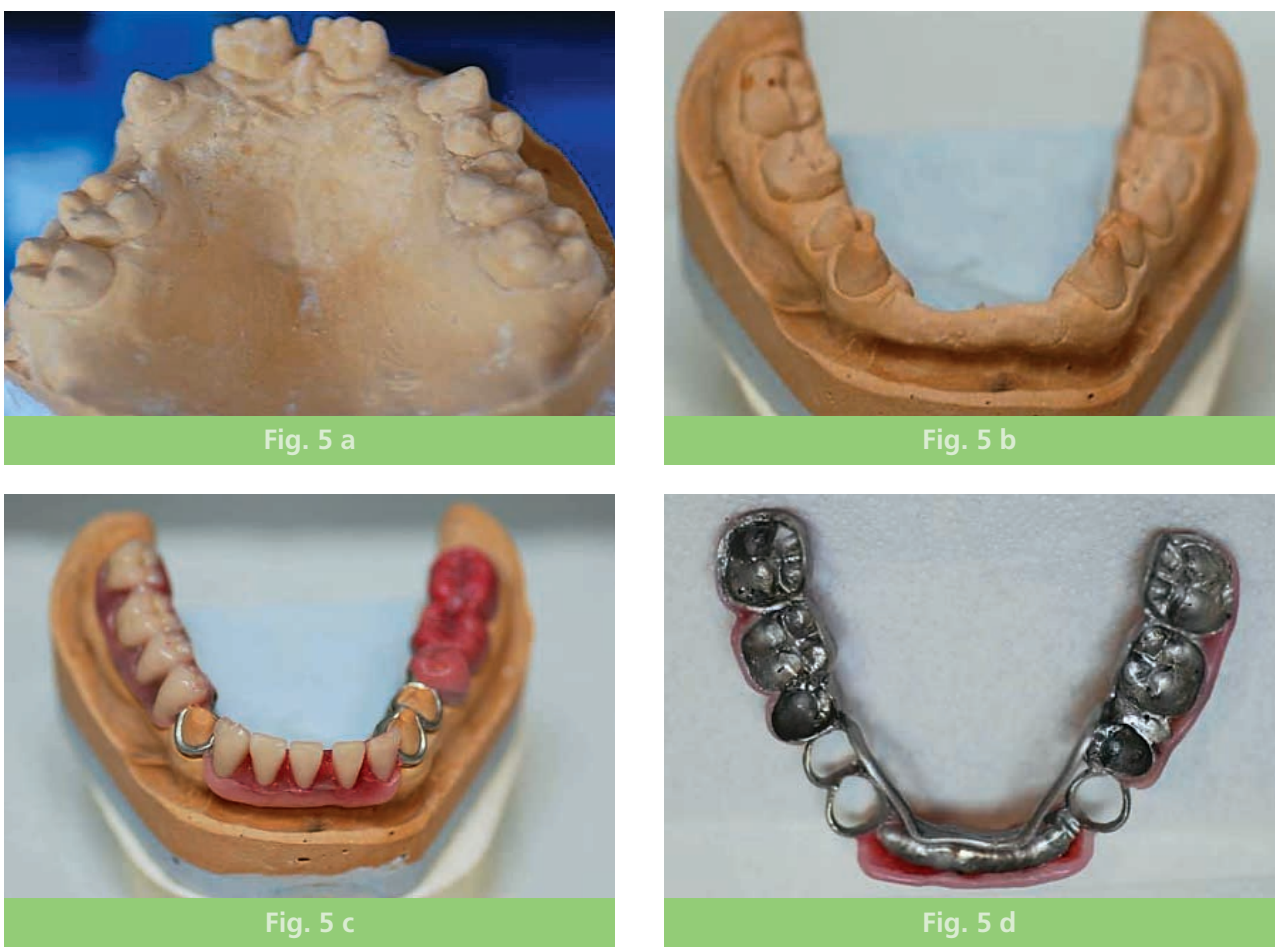

Fig. 5 a à d Situation initiale à 25 ans.

a et $b$ : modèles en plâtre maxillaire et mandibulaire ; $\mathrm{c}$ : prothèse mandibulaire (châssis métallique et des dents sur cire); $d$ : extrados de la prothèse. 
daire est réalisée au Permlastic ${ }^{\circledR}$. L'enregistrement de l'occlusion et le montage en articulateur des modèles de travail ont été réalisés selon les règles classiques de prothèse amovible. La dimension verticale retenue est celle déterminée par le contact dento-dentaire entre 23 et 33 . Les châssis, tant maxillaire que mandibulaire à recouvrement, sont validés lors de l'essayage en bouche. La prothèse est réalisée en un alliage chrome-cobalt-molybdène (Vitallium) (fig. 5 a, 5 b, 5 c et $\mathbf{5}$ d). La concep- tion de la prothèse vise à ménager le parodonte des dents résiduelles, en dégageant notamment le plus possible les embrasures aux alentours des piliers.

Un suivi régulier du patient a été effectué afin de s'assurer de la bonne adaptation des prothèses et de réaliser les corrections nécessaires. Les prothèses amovibles donnent toute satisfaction au patient qui a été informé de la possibilité de réhabilitation ultérieure par prothèse implanto-portée (fig. 6 a à 6 f et fig. 7 a à 7 c).
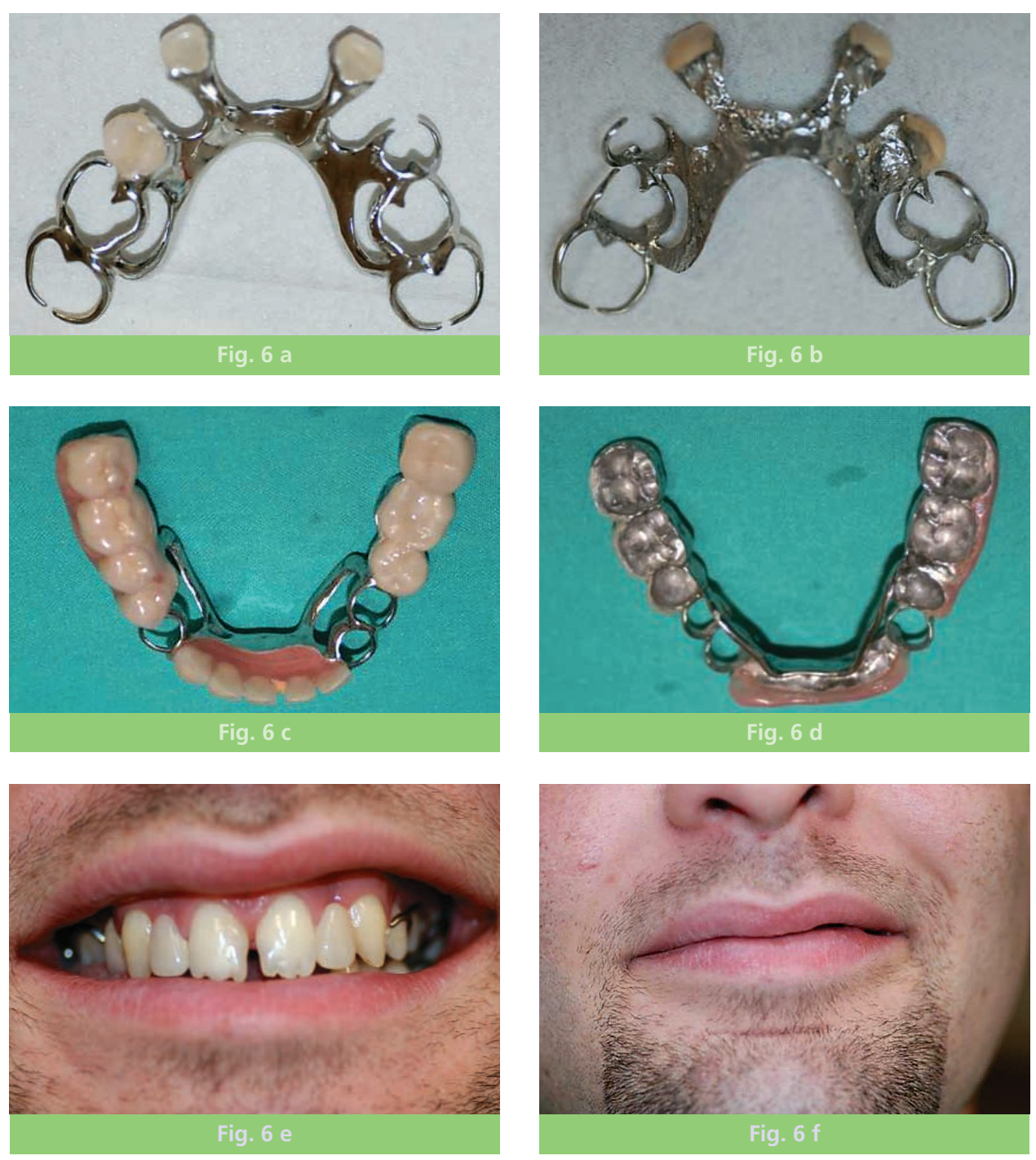

Fig. $6 \mathrm{a}$ à $\mathrm{f}$

$a$ à $d$ : prothèses finies. Différentes vues des prothèses; e et $f$ : prothèse in situ. 

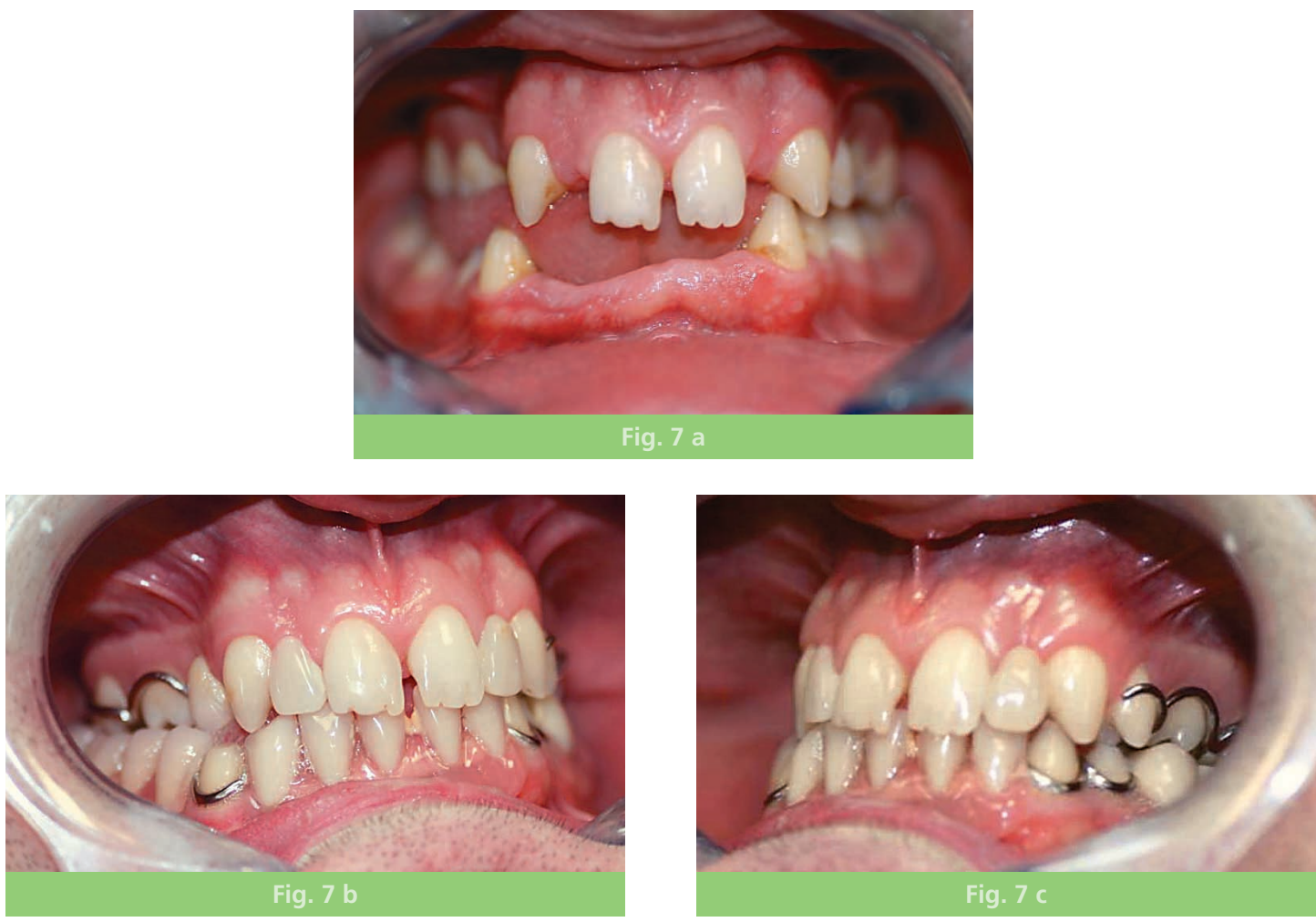

Fig. 7 a à c Résultat final.

a : situation initiale ; b : vue de profil droit, prothèse in situ ; : vue de profil gauche, prothèse in situ.

La prothèse à recouvrement a permis de compenser artificiellement l'espace important situé entre les arcades suite aux malpositions des organes dentaires en rapport avec la dysplasie ectodermique. De plus, cette réhabilitation s'effectue sans que les dents subissent la moindre "mutilation». Les contacts au niveau des canines du côté gauche se sont avérés utiles pour l'enregistrement des relations intermaxillaires, la DVO ainsi déterminée ne paraissant pas pathologique.

La complexité de la prise en charge sur le plan bucco-dentaire des patients atteints de syndrome ou d'affection rares nécessite une collaboration étroite avec différents spécialistes: ORL, dermatologue, chirurgien plastique, médecin. II n'existe aucun schéma reproductible de traitement. Le choix de la modalité thérapeutique est fonction de l'âge et des anomalies stomatognatiques du patient: prothèses, orthodontie, chirurgie orthognatique, mise en place d'implants. La prise en charge doit toutefois être précoce; cela suppose une formation des professionnels, voire l'orientation des patients vers des centres spécialisés. Le diagnostic précoce permet en effet d'orienter la famille, de mettre fin à d'interminables interrogations relatives à l'affection et d'éviter au maximum les problèmes psychoaffectifs et relationnels. Les désirs et les exigences des patients restent un critère décisif pour l'acceptation et le suivi du traitement. 


\section{Bibliographie}

1. Ahmed B, Yazdanie N. Hypohydrotic ectodermal dysplasia (HED).

J Coll Physicians Surg Pak 2006;16(1):61-63.

2. Anoop TM, Simi S, Mini PN, Ramachandran M, Jabbar PK, Rajakumari PK et al.

Hypohydrotic ectodermal dysplasia.

J Assoc Physicians India 2008;56:268-270.

3. Pipa Vallejo A, Lopez Arranz Monje E, Gonzalez Garcia M, Martinez Fernandez M, Blanco Moreno Alvarez Buylla $F$.

Treatment with removable prosthesis in hypohidrotic ectodermal dysplasia. A clinical case. Med Oral Pathol Oral Cir Bucal 2008;13(2):E119-123.

4. Rad AS, Siadat $H$, Monzavi A, Mangoli AA. Full mouth rehabilitation of ectodermal dysplasia patient with dental implants: a clinical report.

J Prosthodont 2007;16(3):209-213.

5. Yavuz I, Kiralp S, Baskan Z. Hypohidrotic ectodermal dysplasia: a case report. Quintessence Int 2008;39(1):81-86.

6. Marques Da Costa $C$, De La Brassinne M. Les dysplasies ectodermiques. Rev Med Liege 2003;58(1):37-40.

7. Chami B, Rahmani EM, Naoumi N, Hafid A, El Houri M,
Mohtarim B, et al. La dysplasie ectodermique anhidrotique :

À propos de deux cas. Actualités Odonto-Stomatologiques 2007;237:83-92.

8. Kramer FJ, Baethge C, Tschernitschek $\mathrm{H}$. Implants in children with ectodermal dysplasia: a case report and literature review. Clin Oral Implants Res 2007;18(1):140-146.

9. Pavlov M, Artaud C, Naulin-Ifi C.

Étude d'un cas de dysplasie ectodermique $\mathbf{X}$ dépendante. Actual Odonto-Stomatol (Paris) 1998;203:367-76.

10. Tarjan I, Gabris K, Rozsa N. Early prosthetic treatment of patients with ectodermal dysplasia: A clinical report. J Prosthet Dent 2005;93:419-424.

11. NaBadalung DP. Prosthodontic rehabilitation of an anhidrotic ectodermal dysplasia patient: a clinical report.

J. Prosthet Dent 1999;81:499-502.

12. Badre B, Kaoun $K$, Bousfiha B, Bousfiha A, Msefer S.

La dysplasie ectodermique anhidrotique.

À propos d'un cas clinique. Journal Dentaire du Québec février 2003;vol 40:69-73.

13. Bal C, Bal B.T, Tüfekcioglu D. Treatment considerations for a patient with hypohidrotic ectodermal dysplasia: a case report. J Contemp Dent Pract 2008;9(3):128-134.

14. Correa D, Carro A, Lopez M. Anodontie associée à une dysplasie ectodermique anhidrotique. À propos d'un cas clinique. Rev Odonto Stomatol 2000;29(1):17-22.

15. Hickey AJ, Vergo TJ. Prosthetic treatments for patients with ectodermal dysplasia. J Prosthet Dent 2001;4(86):364-8.

16. Hickey AJ, Salter M. Prosthodontic and psychological factors in treating patients with congenital and craniofacial. defects. J Prosthet Dent 2006;95(5):392-6.

17. Kearns G, Sharma A, Perrot D, Schmidt B, Kaban L, Vagervik K. Placement of endosseos implants in children and adolescents with hereditary ectodermal dysplasia.

Oral Surg Oral Med Oral Pathol Oral Radiol Endod 1999;88:5-10.

18. Kiener $\mathrm{P}$, Otterli $\mathrm{M}$, Mericske-Stern R. Réhabilitation prothétique des patients atteints de dysplasie ectodermique. Présentation d'un cas.

Rev Mens Suisse Odontostomatol 2003;113:1085-1089.

19. Naser B.

La prothèse adjointe totale et subtotale chez les enfants atteints 
de dysplasie ectodermique (anodontie, oligodontie). Rev Odonto Stomatol (Paris) 1997;26(1-2):123-131.

20. Pavarina $A C$, Machado $A L$, Vergani CE, Giampaolo ET. Giampaolo ET.

Overlay removable partial dentures for a patient with ectodermal dysplasia: A clinical report. J Prosthet Dent 2001;86(6):574-7.

21. Pigno MA, Blakman RB, Cronin RJ, Cavazos E. Prosthodontic management of ectodermal dysplasia: A review of the literature. J Prosthet Dent 1996;76:541-5.

22. Artis JP, Artis $M$, Cassang $S$. Conséquences dentaires et maxillo-faciales de la dysplasie ectodermique anhidrotique.
Actual Odonto-Stomatol (Paris) 1992;180:773-88.

23. Bergendal B, Ekman A, Nilsson $\mathrm{P}$. Implant failure in children with ectodermal dysplasia: a retrospective evaluation of use and outcome of dental treatment in children in Sweden. Int J Oral Maxillofac Implants 2008;23(3):520-524.

24. Bonin B, Saffarzadeh A, Picard A, Levy P, Romieux G, Goga D.

Traitement implantaire précoce d'un enfant atteint d'une dysplasie ectodermique anhidrotique. À propos d'un cas. Rev Stomatol Chir Maxillofac 2001;102(6):313-318.

25. Stanford CM, Guckes $A$, Fete M, Richter MK. Perceptions of outcomes of implant therapy in patients with ectodermal dysplasia syndromes. Int J Prosthodont 2008;21(3):195-200.

26. Sharma A.B, Vargervik K. Using implants for the growing child. J Calif Dent Assoc 2006;34(9):719-724.

27. Castellanos J, Correa D, Carro A, Lopez M.

Anodontie associée à une dysplasie ectodermique anhidrotique. À propos d'un cas clinique. Rev Odonto Stomatol (Paris) 2000;29(1):17-21.

28. Windchy AM, Morris JC. An alternative treatment with the overlay removable partial denture: A clinical report. J Prosthet Dent 1998;79:249-53.

\section{SUMMARY}

\section{Ectodermal dysplasia : prosthodontic rehabilitation clinical report}

Yomin Cécile ALLOH AMICHIA, Aboudramane BAMBA, Laurent LE GUÉHENNEC, Pascal BOURDEAUT, Bernard GIUMELLI

\footnotetext{
Keywords

- anhidrotic ectodermal dysplasia

- Christ-Siemens-Touraine syndrome

- hypodontia or anodontia

- hypohidrosis or anhidrosis

- hypotrichosis

- prosthetic rehabilitation
}

Hypohidrotic ectodermal dysplasia, with an X-linked inheritance, is the most frequent form of ectodermal dysplasias. Ectodermal dysplasia is characterized by a triad of clinical signs: hypohidrosis or anhidrosis (lack of sweat glands), hypotrichosis (scanty, fine light hair on the scalp and eyebrows), hypodontia or anodontia (partial or total decrease of teeth number).

The absence of teeth may constitute functional and aesthetical disability involving a prosthetic rehabilitation. For psychosociological reasons, this rehabilitation is required as soon as possible in young children.

This clinical report describes the different steps of prosthetic rehabilitation in a young man aged 25 suffering from ectodermal dysplasia (ChristSiemens-Touraine syndrome). 